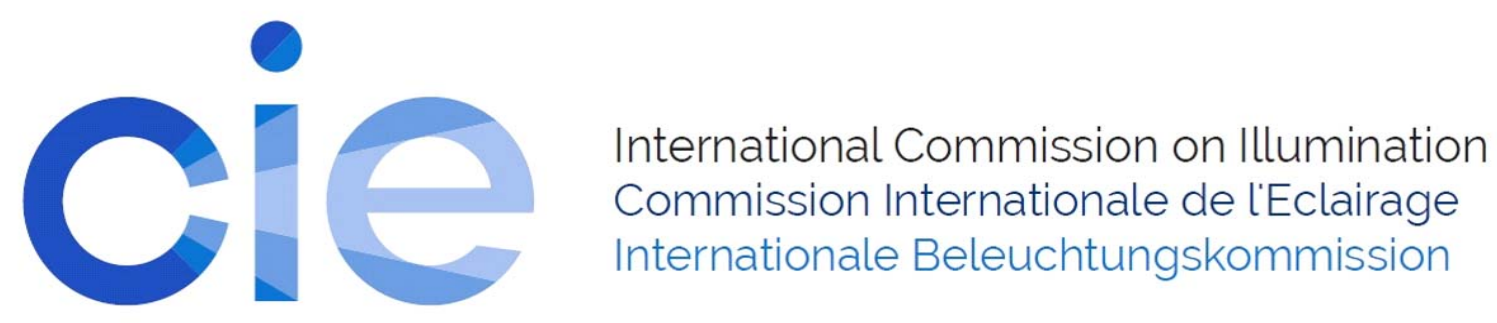

P0192

INNOVATIVE DESIGN AND METROLOGICAL APPROACHES TO SMART LIGHTING

Fabrizio Valpreda et al.

DOI 10.25039/x46.2019.PO192

from

CIE x046:2019

Proceedings

of the

29th CIE SESSION

Washington D.C., USA, June 14 - 22, 2019

(DOI 10.25039/x46.2019)

The paper has been presented at the 29th CIE Session, Washington D.C., USA, June 14-22, 2019. It has not been peer-reviewed by CIE.

(C) CIE 2019

All rights reserved. Unless otherwise specified, no part of this publication may be reproduced or utilized in any form or by any means, electronic or mechanical, including photocopying and microfilm, without permission in writing from CIE Central Bureau at the address below. Any mention of organizations or products does not imply endorsement by the CIE.

This paper is made available open access for individual use. However, in all other cases all rights are reserved unless explicit permission is sought from and given by the CIE.

CIE Central Bureau

Babenbergerstrasse 9

A-1010 Vienna

Austria

Tel.: +4317143187

e-mail: ciecb@cie.co.at

www.cie.co.at 


\title{
INNOVATIVE DESIGN AND METROLOGICAL APPROACHES TO SMART LIGHTING
}

\author{
Valpreda, F. ${ }^{1}$, lacomussi, P. ${ }^{2}$, Rossi, G. ${ }^{2}$ \\ ${ }^{1}$ Politecnico di Torino, Torino, ITALY, ${ }^{2}$ INRIM, Torino, ITALY \\ fabrizio.valpreda@polito.it
}

DOI 10.25039/x46.2019.PO192

\begin{abstract}
Currently Smart Lighting is mostly oriented toward energy savings and road luminance control to acknowledge actual maintenance, weather and traffic conditions, with the target to satisfy drivers visual needs. Alternative applications of smart lighting are toward city beautification and do not have normative constraints toward users safety and visibility as instead are required for road lighting. The paper present two case study of smart lighting systems for $\mathrm{C}$ lighting class road, outcome of a project set up by Politecnico di Torino and INRIM aimed to define new approaches to design smart controls consistent with user's and society needs and to define and assess their metrological performances and calibration requirements.
\end{abstract}

Keywords: Smart lighting, Road lighting, ILMD detector, Pedestrian safety, Systemic Design, Design by Components, Design Network, SURFACE, EMRP

\section{Scenario description}

Smart lighting is one of the most already implemented field of application of Smart Cities, lighting designers look forward to new applications of smart lighting, but unfortunately, applications are manly focused on two main applications: road lighting control toward energy savings in compliance with available regulations on reduction lighting class modifications, city beautification and spectacularization. This last aspect is mostly related to citizen interaction and touristic improvements and represents an underrating of possibilities of smart devices/detectors and loT (Internet of Things).

Designers and metrologists of the current lighting community are used to the aforesaid applications. Metrology is mostly focused providing calibration (ROSSI 2017) and measurement procedures for the road luminance measurements (ROSSI 2015), because road luminance is the relevant parameters in current normative (CIE 2019) (EN 2015 a to b) to assure safety of road users. Even if for specific road classes, and when the luminance approach cannot be applied due to external constraints (like the viewing distance), can occur that illuminance can be used instead of.

In the past years large energy saving in road lighting were achieved thanks to the change of lighting sources, substituting obsolete lighting system based on discharge lamps, with new LED system. Unfortunately in the next years, energy savings is condemned to decrease. In the future the expected potential saving $(6 \%)$ due only to sources will be very less significant than the big step achieved at the introduction of LED (for the same amount of light of an incandescent source the electrical consumption of a LED source is about one tenth: $200 \mathrm{~lm} / \mathrm{W}$ efficiency emission against $20 \mathrm{~lm} / \mathrm{W}$ of an incandescent lamp). Significant additional savings can be achieved only by Smart lighting Systems specifically designed. But to true implementation of Smart Cities it is necessary to move forward only energy savings and city beautification. This can be achieved only with a new wider design approach based on multidisciplinarity in order to provide lighting community smart lighting applications driven not only to energy savings but also to lighting quality, having always the main goal of increasing road users safety.

This new approach is the one followed by a design methodology developed starting from the end of the last century: Systemic Design. Its strong connections with the holistic view of our lives, is one of the most powerful tools available to define effective solutions in product 
design, production systems and connections between nodes providing different services to local, or even global communities, thus adopting a circular economy approach.

Within these conditions multidisciplinary is one of the most practical approaches: humanities and technicalities were proven to be limited to their own environment. Results obtained by a network of nodes providing specific knowledges connected to others with multi-direction links to the entire network is proven (JONES 2014) to be much more effective and efficient that the sum of the single, isolated nodes. This is the approach followed during a multidisciplinary workshop organized by Politecnico di Torino (PoliTo), dedicated to Smart Lighting. Workshop students are put in charge of a design challenge with a lead designer and other fields scientists, and support of a production company, all involved in the design process from the very beginning.

In the Smart Lighting workshop designers (with competencies on loT and Systemic Design), metrologists of INRIM and technician of REVERBERI Enetec, a company producing road lighting control systems, designed several smart systems for public lighting including their metrological capabilities and procedures for performances verification. Keys strengths of the results are in the aforesaid topics: satisfaction of users' needs based on lighting quality as suggested by European Standard (EN 2015d), circular economy with a comprehensive overview of energy re-use, concurrent definition of metrological procedures to assess measuring capabilities toward a lighting quality assessment as in EN standards, IoT for Smart Lighting for Smart Cities.

\section{The technological environment}

In order to define the technological environment where designers and lighting scientists had been involved in, it is necessary to describe, however, the technical and regulatory work field.

In the last fifteen years large technological advances in lighting engineering emerged: mass production of innovative lighting sources, control devices and design tools based on new scientific findings on SSL (Solid State Lighting) tellingly modified the approach to public lighting and to metrological characterization of devices and systems. Of course, energy savings got the biggest advantages by the introduction of SSL when it started about ten years ago, road lighting standard recognized also the possibility of advance lumen output control of SSL and allowed the design of adaptive lighting. Significant future additional savings can be achieved only by smart lighting systems able to manage adaptive lighting, indeed smart controls are mostly addressed to cut energy consumptions keeping constant the user safety by means of controlling the lighting levels accordingly to users' needs, weather conditions and system maintenance. This last aspect guarantees to road managers the optimization of indirect costs.

The metrological definition of users' needs and its measurement is a challenge for metrology: after all, mesopic approach to road lighting is hardly expendable outside tunnel lighting. Moreover, the technology to design advanced smart control is already available and LED impact, in industrialized countries at least, nowadays is aimed toward not only energy savings, but night-time safety, health and wellbeing thanks to lighting systems based on connectivity and smart controls. But new design paradigms and metric headed to these new objectives are necessary, guaranteeing the same (or increased) safety of road users, increasing the quality of lit environment, therefore there will be no need to measure only energetic and light levels.

Road lighting is an interesting field of application of smart city and lighting quality, where a metrology oriented to quantify peculiarities ascribable only to mere SI units has to merge with the needs of measuring the quality of lighting in strict conjunction with human visual perception, moreover this approach has been acknowledged by European standards in road lighting (EN 2015 b to d). . In road lighting applications, devices based on ILMD (Image Luminance Measuring Device) measure the road surface luminance for controlling adaptive lighting systems and modifying lighting level optimising vision conditions according to standards requirements with the additional values of reducing energy consumption and the environmental impact of the installation. 
Usually adaptive system instrument measures the road surface luminance in the range between $0,1 \mathrm{~cd} \mathrm{~m}^{-2}$ and $3 \mathrm{~cd} \mathrm{~m}^{-2}$ (EN, 2015a) while the measurement distance is about $80 \mathrm{~m}$ (EN, 2015b) but for geometrical and practical reasons (installation at about $5 \mathrm{~m}$ above the road surface) this distance is generally higher in real installations, e.g. up to $250 \mathrm{~m}$ (Rossi, $2015)$, to approximate the condition of an view angle of $1^{\circ} \pm 0,5^{\circ}$ as requested by standard (EN, 2015b) and technical report (CIE, 2001), that considers the availability of luminance coefficient data. Adaptive system are also able to infer weather conditions from the spatial distribution of the light reflected by the road, but for this application the knowledge of how pavements behaves under different weather condition is necessary.

The approach of EN standards (EN 2015 a to d) follows CIE publication 115 (CIE 2010) establishing the relevant values of parameters for each road lighting class. The lighting class of a road is selected at national level, following prescription of (TR 2015), that acknowledge the possibility of adaptive lighting if given conditions arrive. Adaptive lighting is defined in (CEN, 2014) as "temporal controlled changes in luminance or illuminance in relation to traffic volume (e.g. veh/5 min), time, weather or other parameters".

For example adaptive road lighting installations are able to maintain constant, at the requested value, the road surface luminance or illuminance despite the installation ageing or the weather condition. Adaptive lighting is obviously focused to satisfy visual needs of road users but under reduced traffic or under varying weather conditions, with the positive benefits of energy savings at the given safety level stated in the standard (EN 2015 a). Usually adaptive systems are installed in lighting class $M$ roads (motorized) or in front of tunnel: in both cases the road luminance is the controlled parameter.

Additional road environments are the $\mathrm{C}$ and $\mathrm{P}$ lighting class: $\mathrm{C}$ class is the conflict area where vehicle flux intersect or interact with weaker users like pedestrian, $\mathrm{P}$ class is for pedestrian (or pedal cyclist) and low speed vehicles $(<40 \mathrm{~km} / \mathrm{h})$ area. $\mathrm{M}, \mathrm{C}$ and $\mathrm{P}$ class strongly relate each other and, in the urban environment, cannot be considered as separate and isolated environments. Normative (EN 2015a) suggests for $C$ lighting class illuminance values (and uniformity in illuminance too) instead of luminance when "calculations - in luminance - do not apply or are impracticable. This can occur when the viewing distances are less than $60 \mathrm{~m}$ and when several observer positions are relevant". For $\mathrm{P}$ lighting class, analogously requirements on illuminance apply, given that pedestrian visibility needs are different from drivers' needs, not only because different velocity occurs, but also because geometry of observation are different. In these cases, it's obvious that smart system based on ILMD absolutely needs a deep knowledge of road surface behavior in order to link luminance measurements to illuminance values. Currently this behavior is described by luminance coefficient, $q$, the ratio between luminance and illuminance for given directions of illumination and for single direction of observation. that -currently- is similar to car drivers' condition of observation. These geometrical conditions are defined in r-tables (CIE 2001) together with some reference data of luminance coefficient of selected pavements made in the seventies. Obviously these data are not suitable for smart systems that for practical reasons observe the road, or a lit environment, from completely different point of view, but as well for road lighting design and road users, because refer to old pavements, and technology and materials of road surfaces evolved a lot in the last 40 years. Stating this it is clear the inherent difficulty of using the current reference data to design and adapt a lighting set up according to users' needs. In this metrological framework, calibration on mere SI units is not effective. Smart materials and loT approaches can help building reference calibration procedures and Reference Materials* (RM) for the given calibration quantities and conditions.

"material sufficiently homogeneous and stable with respect to one or more specified properties (qualitative or quantitative), which has been established to be fit for its intended use in a measurement process" (ISO 2007) 


\section{Case Studies}

Usually smart systems are designed for $\mathrm{M}$ lighting class roads and road tunnel, and are able to:

- Select the correct lighting class according to the traffic volume,

- Detect weather conditions,

- Maintain the road luminance equal to the maintained average value required by standard for the given lighting class,

- Detect anomalous situation like the presence of car queues and wet road surface,

- For tunnel, detect the luminance threshold and adapt tunnel entrance lighting according.

Given that Smart City is based on connectivity not only in term of data management, but also of users needs having in the future plenty of possible applications, the workshop of PoliTo and INRIM head toward different urban environments than M lighting class roads or tunnel, this is the first results of the aforementioned multidisciplinary approach. The case studies hereafter described focus on applications for conflict area ( $C$ lighting class) where the weakest users are pedestrian. Both studies consider safety-related issues of the pedestrian detection by drivers in correspondence of crossing. The design concept is based upon the following guidelines:

- Visibility, traffic and weather data gathering, also according to WMO future approach of diffuse data gathering in towns, the so called Urban Integrated Services (WMO 2018);

- Data analysis and communication

- Technology choice

- Energy management and reuse of savings provided by adaptive lighting and SSL

- Additional active signalling to increase pedestrian visual conspicuity

The first case considers pedestrians crossing in adverse visibility conditions. The result is a system of sensors connected to a data-cloud that gathers and distributes information about pedestrian position and movement while crossing road. The system uses those data to manage pedestrian crossing, either stopping approaching cars, or preventing people to cross the street, depending on traffic conditions. What is relevant in this case study is the sensors system, used to detect and measure weather conditions including fog and atmospheric turbidity, and how those measurements are used on field.

The second case study is quite similar to the first one, given that it provides data gathering and measurement of vehicles traffic and lighting on field. Those information, however, are used to improve the vision of the pedestrian approaching the streets from the point of view of the vehicles. The result is a system that provides a more accurate, detailed and therefore safe illumination through an adaptive lighting system, able to increase road luminance (and illuminance) to higher lighting class requirements, according to pedestrians' position and movement in real time, adding active signalling too.

\subsection{Metrological requirements}

Given that both systems need to detect pedestrian presence, the main metrological challenges are in measuring the quantities of interest or needed for calculations. For the first device the quantities of interest are the atmospheric turbidity, extinction coefficient and wavelength absorption, and/or related climate/pollution quantities measured by additional detectors that can be installed upon request.

Calibration can be done in lab as well on site, because the periodicity of the installation that is in conjunction with the lighting poles, calibration can be done in relative units too. The possible implementations are towards WMO requirements (WMO 2018) on climate related parameters measured on site, after laboratory calibration. 
The second device is the one of highest interest for the lighting community: the system must measure on site luminance and illuminance of the conflict area of pedestrian crossing, so it is based on an ILMD detector. Indeed (EN 2015a) and (TR 2015) suggest that for C lighting class, the quantity of interest can be also illuminance when is hard to define luminance because geometrical constraints like viewing distances. Road luminance is linked to road illuminance by the luminance coefficient $q$.

$$
q=\frac{L}{E}
$$

Where $L$ is the luminance of the road and $E$ the illuminance. The values of $q$ are currently published on reference tables, called r-table (CIE 2010), but unfortunately, the data refer to no more actual pavements (DUMONT 2006) (FOTIOS 2007) and for useless geometries e.g. the condition of observation is $1^{\circ}$, that corresponds to viewing distance higher than $60 \mathrm{~m}$ that is not suitable for the geometrical installation of the ILMD close to the pedestrian crossing. As well the direction of illumination of the crossing field are different from the ones in CIE rtables.

Usually ILMD calibration is made in luminance according to several simplifications on the road surface reflectance behaviour or with on-site procedures. In this case the geometry of luminance measurement is different from the geometry of observation of driver, and the measured luminance can be different from the perceived one. But not only: to measure the illuminance on the road, the tabulated values of $q$ are unusable, because current pavements are different from the tabulated ones, but especially the geometry of interest is different. So, the knowledge of the road surface luminance coefficient for different geometries is of fundamental importance, as well the spectral behaviour of pavement (radiant values of $q$ ), of marking and their interaction with the installed SSL. These are the expected outcomes of the project Surface that was involved in the workshop of PoliTo and INRIM too. Moreover as highlighted in a recent study [Gidlund 2019] the use of bright pavements can provide relevant advantages in energy savings, but also in pedestrian visibility and comfort. In this case the relevance of the knowledge of radiant q quantity and ILMD spectral sensitivity play a relevant role.

\section{Acknowledgement}

This work is partially covered by project "16NRM02 Surface, Pavement surface characterisation for smart and efficient road lighting" that has received funding from the EMPIR programme. EMPIR programme is co-financed by the Participating States and from the European Union's Horizon 2020 research and innovation programme.

Authors are grateful to REVERBERI Enetec Smart Solution for contributions to the workshop.

\section{References}

CIE 2001 CIE144:2001. Road Surface and Road Marking Reflection Characteristics; Technical Report. Vienna: CIE.

CIE 2010 CIE115:2010. Lighting of Roads for Motor and Pedestrian Traffic; Technical Report; Vienna: CIE.

CIE 2011. CIE 194:2011. On Site Measurement of the Photometric Properties of Road and Tunnel Lighting. Vienna: CIE.

CIE 2019. CIE 140:2019 Road lighting calculations. Technical Report. Vienna: CIE.

DUMONT E.; 2007 Paumier, J. Are standard tables R still representative of the properties of road surfaces in France? Proc. of the CIE 26th Session, Beijing;

EN 2015a EN 13201-2:2015. Part 2: Performance requirements. In Road Lighting; CEN: Bruxelles: CEN. $\square$ 
EN 2015b EN 13201-3:2015. Part 3: Calculation of performance. In Road Lighting; CEN: Bruxelles: CEN.

EN 2015c EN 13201-4:2015. Part 4: Verification of performance. In Road Lighting; CEN: Bruxelles: CEN.

EN 2015d EN 13201-5:2015. Part 5: Energy performance indicators. In Road Lighting; CEN: Bruxelles: CEN.

FOTIOS, S. 2006; Boyce, P.; Ellis, C. The effect of pavement material on road lighting performance. Light. J. Rugby $\square, 71,35$.

GIDLUND, H. 2019 et al., Road Surface Photometric Characterization and Its Impact on Energy Savings, Coatings, 9(5), 286; doi:10.3390/coatings9050286

ISO 2007 ISO/IEC Guide 99:2007, International vocabulary of metrology - Basic and general concepts and associated terms (VIM)

JONES, P. H. 2014. Systemic Design Principles for Complex Social Systems. Social Systems and Design, Translational Systems Sciences, vol. 1, pp. 91-128. Tokyo: Springer Japan

ROSSI, G. 2015 et. al. Adaptive Systems in Road lighting installations. Proceedings of the 28th Session of the CIE, Manchester 2015.

ROSSI, G. 2017 et. al. Metrological characterization of ILMD for smart lighting applications. Proceedings of the CIE, Jeju

TR 2015, TR-EN 13201-1:2015. Part 1: Selection of road classes. In Road Lighting; CEN: Bruxelles, Belgium, 2015.EN 13201-2:2015. Part 2: Performance requirements. In Road Lighting; CEN: Bruxelles.

WMO 2018, WMO Guide for Urban Integrated Hydro-Meteorological, Climate and Environmental Services, WMO. Available at https://www.wmo.int/pages/prog/arep/gaw/documents/UrbanIntegratedServicesPart1aCon ceptandMethodologyEC-70.pdf (retrieved on April 15) 